\title{
Triage and Outcomes for a Whole Cohort of Patients Presenting for Major Emergency Abdominal Surgery Including the No-LAP Population: A Prospective Single-Center Observational Study
}

\section{Mohamed Ebrahim ( $\sim$ mohamed.ebrahim.01@regionh.dk)}

Department of Gastrointestinal Surgery, Hvidovre Hospital, University of Copenhagen, Copenhagen, Denmark

\section{Morten Laksáfoss Lauritsen}

Department of Gastrointestinal Surgery, Hvidovre Hospital, University of Copenhagen, Copenhagen, Denmark

\section{Mirjana Cihoric}

Department of Anesthesiology and Intensive Care Medicine, Hvidovre Hospital, University of Copenhagen, Copenhagen, Denmark

\section{Karen Lisa Hilsted}

Department of Gastrointestinal Surgery, Hvidovre Hospital, University of Copenhagen, Copenhagen, Denmark

\section{Nicolai Bang Foss}

Department of Anesthesiology and Intensive Care Medicine, Hvidovre Hospital, University of Copenhagen, Copenhagen, Denmark

\section{Research Article}

Keywords: Surgery, Perioperative Medicine, Mortality, Frailty, Patient Selection

Posted Date: March 8th, 2022

DOI: https://doi.org/10.21203/rs.3.rs-1421139/v1

License: (c) (1) This work is licensed under a Creative Commons Attribution 4.0 International License. Read Full License 


\section{Abstract}

\section{Background:}

Clinical outcomes for patients with an indication for major emergency abdominal surgery, who do not proceed to the operating room, are not well characterised in the existing literature. Triage criteria may vary between centres, which could significantly impact reported postoperative outcomes.

\section{Methods:}

We aimed to characterise the entire population presenting with an indication for acute high-risk abdominal surgery, including patients not proceeding to surgery, in a prospective observational singlecenter study in a high-volume Danish center.

In all, 252 consecutive patients presenting with acute surgical abdominal pathology, where major emergent abdominal surgery was indicated, were prospectively included from $15^{\text {th }}$ October 2020 to $15^{\text {th }}$ August 2021.

\section{Results:}

Overall, 21 patients $(8.3 \%)$ of our total study cohort did not proceed to surgery. These patients were significantly older, more comorbid with higher ASA scores, poor performance status, and were more likely to have bowel ischemia. Poor functional performance and surgeons' consideration of futile intervention were the main reasons for the decision not to operate in all 21 patients. Overall, 30-day mortality was $95 \%$ for the No-LAP cohort, $9 \%$ for the LAP cohort, and $16 \%$ for the whole cohort respectively. Thus, indicating the No-LAP group selection as one of the main determinants of overall outcome and postoperative reported outcome.

\section{Conclusions:}

There is a need for prospective international multi-center studies to characterise the entire cohort of patients eligible for emergency laparotomy including the No-LAP population, as large variations in triage criteria and culture seem to exist.

Trial registration: Retrospectively registered.

\section{Background}

While acute high-risk abdominal surgery (AHA) surgery, defined as major emergency abdominal pathology requiring emergency laparotomy or laparoscopy can be lifesaving, it carries a considerable risk of poor outcomes. Especially in high-risk patients, surgery may be deemed futile. International data indicate that there potentially are significant variations in triage criteria[1-6]. The decision to operate frail patients presenting with major acute surgical abdominal pathology is challenging and is affected by 
multiple factors including patient characteristics, surgeon's experience, intuition, cultural differences in healthcare systems, and the patients' beliefs and values[1,7-9]. Although several studies have assessed outcomes following emergent major abdominal surgery in frail patients, and different mortality and morbidity prediction systems have been developed to support clinical decision making[10, 11], only one prospective study has prospectively reported outcomes for patients who fulfil criteria for surgery but do not proceed to surgery ( The No-LAP population)[12]. This recent single-center study showed that the NoLAP population represented $32 \%$ of all patients meeting the criteria for emergency laparotomy/laparoscopy according to the NELA ( National Emergency Laparotomy Audit) criteria[12]. While this population is prevalent in daily clinical practice, the cohort, in general, is not well described in current studies and audits. The lack of data complicates the comparison of outcomes for the whole cohort and challenges rational conclusions to be drawn on the relative outcome e.g. mortality, including effects of patient care pathways, as well as the establishment of evidence-based guidelines to define futility and guide decision-making in surgery. [6].

We aimed to characterise the entire population fulfilling criteria for AHA surgery in a single Danish center, with a well-established patient pathway, to evaluate potential differences in surgical triage culture, as well as the impact of preoperative decision-making on overall outcomes for the whole population.

\section{Methods}

\section{Approvals}

Permission for the data acquisition for the study was granted by the Center for Regional Development, Capital Region of Denmark (ID no. H-20036076) The Regional Ethics committee had no objections to the study (no. H-20036076) and did not require informed consent from the patients as the study was purely observational. The study was reported according to The Strengthening the Reporting of Observational Studies in Epidemiology (STROBE) guidelines [13]

\section{Patients}

This study was a single-center prospective observational cohort study and included all adult patients $(\geq$ 18 years ) eligible for acute-high risk abdominal surgery (AHA) defined as emergency laparoscopy or laparotomy at the Gastro Unit, Hvidovre University Hospital, Hvidovre, Denmark from October 15th, 2020 to August 15th, 2021, due to the following conditions: (perforated viscus, intestinal obstruction, intestinal ischemia, intraabdominal bleeding, emergency reoperations after elective surgery due to a post-operative complication e.g. intraabdominal bleeding, obstructive ileus, intestinal ischemia or anastomotic leakage). These patients are characterized as AHA (Acute High-risk Abdominal surgery) patients. Patients undergoing the following procedures were excluded: Trauma, appendectomies, cholecystectomies, negative diagnostic laparoscopies/laparotomies, herniotomies without bowel resection, internal herniation after Roux-en-Y gastric bypass, gynecological, urogenital or vascular pathology requiring surgery and subacute surgery ( surgery planned within 48 hours) (Fig. 1) 
Our department serves an area of 515000 inhabitants with approximately 3-400 AHA operations annually[2]. All patients who are suspected to be an AHA-patient undergo a pre-operative care bundle protocol, which includes early preoperative administration of high-dose intravenous broad-spectrum antibiotics, placement of a nasogastric tube and urinary catheter, preoperative blood sample collection, blood and urinary culture analyses, arterial blood gas evaluation and performance of early contrastenhanced abdominal computed tomography (CT), followed by early multidisciplinary evaluation by a consultant anesthesiologist and consultant surgeon[2].

Post-operatively, AHA-patients are either admitted directly to the intensive care unit (ICU) or to the perioperative unit based on the patients general condition and surgical APGAR score[14]. Perioperative unit stay length is determined by the surgical APGAR score. Patients are discharged from the perioperative unit to a specialized subunit in our surgical department where bedside rounds are caried daily by a dedicated team of acute care surgeons (senior consultants/consultants). The AHA patients follow an enhanced recovery after surgery (ERAS)-program including multimodal analgesia, early mobilization and oral nutrition, early removal of drains, nasogastric tubes and urinary catheters, lung physiotherapy and follow specific discharge criteria.

The "No-LAP" population was defined as patients who fulfilled the AHA criteria for surgery but did not proceed to surgery. In case of refrainment from surgery, the reasons for the decision were registered. The decisions were categorized before the start of the study and were assessed by the attending surgeon as either patients' choice of declining surgery; surgery likely to be futile either due to poor patient fitness or advanced malignancy with low life expectancy.

Screening of potential NoLap patients was performed daily by the study team and all physicians at our surgical department were informed of the study.

When a patient was considered a NoLAP candidate after evaluation for surgery, the doctor on call contacted the study team. The study team did not guide decision-making as the study was purely observational.

\section{Data collection}

Study data were collected and managed using REDCap electronic data capture tools hosted at Gastrounit, Copenhagen University Hospital Hvidovre[15]. The following data were collected: Age, Sex, American Society of Anesthesiologist (ASA) score, patient comorbidities, Eastern Cooperative Oncology Group (ECOG) performance score[16], indication for surgery, preoperative radiology and findings; charge of deciding surgeon; baseline blood tests including creatinine, eGFR, CRP, hemoglobin, serum albumin, and arterial blood gas were also registered.

\section{Primary and secondary outcomes}

The primary endpoint for this study was to estimate the prevalence of No-LAP patients in a Danish singlecenter setting. Secondary outcomes included 30 and 90 -days mortality for the LAP, No-Lap populations, 
and overall mortality.

\section{Statistical analysis}

Continuous data are presented as median with interquartile range (IQR) and categorical data as number and percentage. When comparing groups, Fisher's exact test was used for categorical variables and the Mann-Whitney test for continuous variables. Kaplan-Meier curves were computed to estimate survival probabilities for the No-LAP and the LAP group. The difference in survival time between the two groups was assessed by the log-rank test. All tests were two-tailed and were conducted at a $5 \%$ significance level and $95 \%$ confidence intervals $(\mathrm{Cl})$ were estimated when appropriate.

All analyses were performed with R statistical software 4.1.2[17]

\section{Power analysis}

A sample size estimation was carried out based on the No-LAP population incidence of $32 \%$ reported by Mcllveen et al[12]. We wished to power the study to detect a relative deviation of more than $25 \%$ in the incidence of NoLap, compared to the previously reported No-LAP population. Using an alpha-cut-off value of $5 \%$ and a beta-cut-off of $20 \%$, a sample size of 253 patients in total, including NoLap patients, was required.

The study was performed with monthly patients counts, so that the study would be terminated on the first full month after the required 250 patients were included. Study termination was therefore assessed on a month-by-month basis pending inclusion of at least 250 patients.

\section{Results}

\section{Patient characteristics}

A total of 252 patients with a median age of 68 years were included in the study (Fig. 1) \& Online Supporting Infortmation Table S1. Overall, 21 (8.3\%), 95\% Cl (5.5\%-12.4\%) patients of our study cohort did not proceed to surgery (NoLAP-patients). The most prevalent indications for surgery were intestinal obstruction, intestinal perforation, and intestinal ischemia which accounted for $57 \%, 30 \%$, and $6 \%$ respectively (Table 1). Patients who did not proceed to emergency surgery were significantly older, more comorbid with higher ASA scores, poor performance status, and were more likely to present with bowel ischemia in contrast to patients who underwent surgery (LAP-population) (Table 1). The No-LAP-patients presented with significantly lower albumin and eGFR levels, and higher CRP, and lactate values as compared with the LAP-population preoperatively. Lower median haemoglobin levels were also observed in the No-LAP group however it did not reach statistical significance $p=0.076$ (Table 1). 
Table 1

Baseline patient characteristics and mortality outcomes

\begin{tabular}{|c|c|c|c|c|}
\hline & $\begin{array}{l}\text { All patients } \\
\mathrm{N}=252\end{array}$ & $\begin{array}{l}\text { NoLAP- } \\
\text { population } \\
\mathrm{N}=21\end{array}$ & $\begin{array}{l}\text { LAP- } \\
\text { population } \\
\mathrm{N}=\mathbf{2 3 1}\end{array}$ & $\begin{array}{l}\mathrm{P} \text { - } \\
\text { value }\end{array}$ \\
\hline Age, years, (median, IQR) & $68(53-78)$ & $76(73-83)$ & $66(52-76)$ & $\begin{array}{l}< \\
0.001\end{array}$ \\
\hline Male, n (\%) & $127(50)$ & $10(48)$ & $117(51)$ & 0.8 \\
\hline ASA-score & $21(8.3)$ & 0 & $22(9.1)$ & 0.001 \\
\hline I & $101(40)$ & $2(9.5)$ & $99(43)$ & \\
\hline II & $105(42)$ & $14(67)$ & $91(39)$ & \\
\hline III & $22(8)$ & $4(19)$ & $18(7.8)$ & \\
\hline IV & $3(1.2)$ & $1(4.8)$ & $2(0.9)$ & \\
\hline \multicolumn{5}{|l|}{ V } \\
\hline WHO Performance status $>2, \mathrm{n}(\%)$ & $22(8.7)$ & $8(38)$ & $14(6.1)$ & $\begin{array}{l}<.001 \\
0.01\end{array}$ \\
\hline \multicolumn{5}{|l|}{ Past medical history, n (\%) } \\
\hline Former intraabdominal surgery & $132(52)$ & $10(47)$ & $122(53)$ & 0.5 \\
\hline Comorbidity (n\%) & $98(84)$ & $21(100)$ & $77(80)$ & 0.023 \\
\hline Diabetes & $18(7.1)$ & 0 & $18(7.8)$ & 0.3 \\
\hline IDDM & $9(3.6)$ & $3(14)$ & $6(2)$ & \\
\hline \multicolumn{5}{|l|}{ NIDDM } \\
\hline Lung disease & $46(18)$ & $8(38)$ & $38(16)$ & 0.033 \\
\hline $\begin{array}{l}\text { Neurological disease including } \\
\text { cerebrovascular diseases }\end{array}$ & $31(12)$ & $5(24)$ & $26(11)$ & 0.4 \\
\hline Nephropathy & $13(5)$ & $2(9.5)$ & $11(4.7)$ & 0.2 \\
\hline Dementia & $6(2.4)$ & $1(4.8)$ & $5(2.2)$ & 0.4 \\
\hline Malignancy (active or previous) & $53(21)$ & $10(48)$ & $43(19)$ & 0.003 \\
\hline Hypertension & $85(34)$ & $9(43)$ & $76(33)$ & 0.4 \\
\hline Atrial Flutter & $26(10)$ & $4(19)$ & $22(9.6)$ & 0.2 \\
\hline
\end{tabular}

IQR, interquartile range; ASA, American Society of Anesthesiology; WHO, World Health Organization; IDDM, Insulin-dependent diabetes mellitus; NIDDM, non-insulin-dependent diabetes mellitus; eGFR, estimated glomerular filtration rate; CRP, C-reactive protein; WBC, white blood cell count. 


\begin{tabular}{|c|c|c|c|c|}
\hline & $\begin{array}{l}\text { All patients } \\
\mathrm{N}=252\end{array}$ & $\begin{array}{l}\begin{array}{l}\text { NoLAP- } \\
\text { population }\end{array} \\
\mathrm{N}=\mathbf{2 1}\end{array}$ & $\begin{array}{l}\text { LAP- } \\
\text { population } \\
\mathrm{N}=231\end{array}$ & $\begin{array}{l}\mathrm{P} \text { - } \\
\text { value }\end{array}$ \\
\hline Heart failure & $6(2.38)$ & 0 & $6(2.6)$ & $>0.9$ \\
\hline Ischemic heart disease & $23(9.2)$ & $3(14)$ & $20(8.7)$ & 0.4 \\
\hline Liver cirrhosis & $7(2.8)$ & $2(9.5)$ & $5(2.2)$ & 0.11 \\
\hline \multicolumn{5}{|c|}{ Blood results at admission, median ( IQR) } \\
\hline Albumin, (g/L) & $32(26-37)$ & $24(20-32)$ & $33(27-36)$ & 0.002 \\
\hline eGFR $\mathrm{ml} / \mathrm{min} /\left(1,73 \mathrm{~m}^{2}\right)$ & $78(52-90)$ & $48(27-83)$ & $78(56-90)$ & 0.004 \\
\hline Potassium (mmmol/L) & $\begin{array}{l}3.9(3.6- \\
4.2)\end{array}$ & $\begin{array}{l}4.20(3.7- \\
4.3)\end{array}$ & $\begin{array}{l}3.9(3.6- \\
4.1)\end{array}$ & 0.10 \\
\hline Creatinine $(\mathrm{mmmol} / \mathrm{L})$ & $82(64-110)$ & $98(64-193)$ & $\begin{array}{l}80(64- \\
103)\end{array}$ & 0.087 \\
\hline Sodium (mmmol/L) & $\begin{array}{l}137(134- \\
140)\end{array}$ & $\begin{array}{l}136.5(131- \\
140)\end{array}$ & $\begin{array}{l}137(134- \\
140)\end{array}$ & 0.6 \\
\hline CRP (mg/L) & $53(9-160)$ & $145(62-222)$ & $48(8-140)$ & 0.015 \\
\hline Hemoglobine (mmmol/L) & $\begin{array}{l}8.10(7.10- \\
9.10)\end{array}$ & $\begin{array}{l}7.25(6.6- \\
8.2)\end{array}$ & $\begin{array}{l}8.2(7.1- \\
9.1)\end{array}$ & 0.076 \\
\hline WBC $\left(\right.$ WBC $\left.\times 10^{9} / \mathrm{L}\right)$ & $\begin{array}{l}10.7(8.7- \\
14.3)\end{array}$ & $10(7-18)$ & $11(9-14)$ & 0.6 \\
\hline Platelets. (Platelets x 109/L) & $\begin{array}{l}295(234- \\
294)\end{array}$ & $\begin{array}{l}266(224- \\
386)\end{array}$ & $\begin{array}{l}266(224- \\
386)\end{array}$ & 0.9 \\
\hline Lactate $>2 \mathrm{mmol} / \mathrm{L}$ & $18(7.1)$ & $7(33)$ & $11(4.8)$ & $\begin{array}{l}<.001 \\
0.001\end{array}$ \\
\hline \multicolumn{5}{|l|}{ Indications for surgery n (\%) } \\
\hline Intestinal obstruction & $145(57)$ & $5(24)$ & $140(60)$ & $\stackrel{<}{<.001}$ \\
\hline Intestinal perforation & $76(30)$ & $8(38)$ & $68(29)$ & 0.7 \\
\hline Intestinal ischemia & $15(6)$ & $8(38)$ & $7(3)$ & $\begin{array}{l}< \\
0.001\end{array}$ \\
\hline Anastomotic leakage & $6(2.4)$ & 0 & $6(2.6)$ & $\begin{array}{l}< \\
0.001\end{array}$ \\
\hline
\end{tabular}

IQR, interquartile range; ASA, American Society of Anesthesiology; WHO, World Health Organization; IDDM, Insulin-dependent diabetes mellitus; NIDDM, non-insulin-dependent diabetes mellitus; eGFR, estimated glomerular filtration rate; CRP, C-reactive protein; WBC, white blood cell count. 


\begin{tabular}{|c|c|c|c|c|}
\hline & $\begin{array}{l}\text { All patients } \\
\mathrm{N}=252\end{array}$ & $\begin{array}{l}\text { NoLAP- } \\
\text { population } \\
\mathrm{N}=\mathbf{2 1}\end{array}$ & $\begin{array}{l}\text { LAP- } \\
\text { population } \\
\mathrm{N}=231\end{array}$ & $\begin{array}{l}\mathrm{P} \text { - } \\
\text { value }\end{array}$ \\
\hline Bleeding & $6(2.4)$ & 0 & $6(2.6)$ & $\begin{array}{l}<.001 \\
0.001\end{array}$ \\
\hline $\begin{array}{l}\text { latrogenic bowel perforation after primary } \\
\text { surgery. }\end{array}$ & $4(1.5)$ & 0 & $4(1.7)$ & $\begin{array}{l}<.001 \\
0.01\end{array}$ \\
\hline \multicolumn{5}{|l|}{ Mortality, n (\%) } \\
\hline 30-day mortality & $41(16)$ & $20(95)$ & $21(9)$ & $<.001$ \\
\hline 90-day mortality & $53(21)$ & $21(100)$ & $32(14)$ & $\begin{array}{l}< \\
0.001\end{array}$ \\
\hline \multicolumn{5}{|c|}{$\begin{array}{l}\text { IQR, interquartile range; ASA, American Society of Anesthesiology; WHO, World Health Organization; } \\
\text { IDDM, Insulin-dependent diabetes mellitus; NIDDM, non-insulin-dependent diabetes mellitus; eGFR, } \\
\text { estimated glomerular filtration rate; CRP, C-reactive protein; WBC, white blood cell count. }\end{array}$} \\
\hline
\end{tabular}

\section{Reasons for deferral from surgery}

Five $(24 \%)$ patients declined surgical treatment due to self-perceived poor performance status and subjective consideration of surgical futility, and the patient's decision was in line with the surgeon's evaluation of probable futility in all cases. Poor performance status and surgeons' consideration of futile intervention were the main reasons for not offering surgery in all 21 patients. An advanced malignancy was a reason for deferral from surgery in $9(43 \%)$ patients.

(Table 2). In all NoLap-patients, the deciding surgeon was either a senior consultant or consultant (Table 2). 
Table 2

Reasons for deferring No-lap patients' surgery.

\begin{tabular}{|c|c|}
\hline Reason why patient was considered No-lap patient, $n$ (\%) & Patient choice $(n=5)$ \\
\hline Patient declined surgery as futile & $5(100)$ \\
\hline $\begin{array}{l}\text { Patient considered their performance status too low to undergo } \\
\text { surgery }\end{array}$ & $5(100)$ \\
\hline \multirow{2}{*}{$\begin{array}{l}\text { Advanced malignancy with low life expectancy considered by } \\
\text { the patient and the surgeon as a main cause to defer from } \\
\text { surgery }\end{array}$} & $1(20 \%)$ \\
\hline & $\begin{array}{l}\text { Indication for futility of surgery as } \\
\text { considered by the surgeon }(\mathrm{n}= \\
\text { 16) }\end{array}$ \\
\hline Poor performance status & $16(100)$ \\
\hline $\begin{array}{l}\text { Advanced malignancy with low life expectancy considered as a } \\
\text { leading cause to defer from surgery }\end{array}$ & $8(50)$ \\
\hline \multicolumn{2}{|l|}{ Charge of deciding surgeon, $n(\%)$} \\
\hline Senior consultant or consultant & $21(100)$ \\
\hline
\end{tabular}

\section{Mortality}

All No-LAP patients but one (95\%), Cl (77.3\%-99.1\%), died within 30-days after admission. The 30-day mortality for the LAP-cohort was $9 \%, \mathrm{Cl}(6.4 \%-13.0 \%)$ and the overall mortality for the entire cohort, including NoLap patients was $16 \%, \mathrm{Cl}(12.2 \%-21.3 \%)$.

All No-LAP patients (100\%), $\mathrm{Cl}(84.5 \%-100 \%)$, were dead 90 days after admission in contrary to the LAPpopulation where 32 patients $(13.85 \%), \mathrm{Cl}(9.98-18.9)$ had died $\mathrm{p}<0.001$. The overall 90 -day mortality rate for the total study cohort was $21 \%, \mathrm{CI}(16.4 \%-26.4 \%)$ (Table 1$)$. The LAP cohort had better survival during the whole study period than the NoLap cohort p-value for log-rank test $<0.001$ (Fig. 2).

\section{Discussion}

This prospective, single-center study aimed to characterise and report clinical outcomes for a consecutive cohort of patients eligible for major emergent abdominal surgery including patients who did not proceed to major emergent abdominal surgery (No-LAP population) in a Danish center with an established care pathway.

The study revealed that $8.3 \%$ of our total patient cohort did not proceed to surgery. This in comparison to the $32 \%$ reported by Mcllveen et al[12]. In our cohort, all but one No-LAP patient (95\%) died within a month after admission while approximately $37 \%$ of the No-LAP patients in the study by Mcllveen et al. were alive 30 days after admission[12] The 30-day mortality rate for the LAP-cohort was $9 \%$ in our cohort 
vs. $12.6 \%$ in the study by Mcllveen et al. However, the 30 -day mortality rate for the whole cohort was $16 \%$ in our study versus $29 \%$ in the data reported previously by Mcllveen et al [12].

Current literature is scarce with only one prospective study reporting outcomes for this patient population $[8,12]$. In our study, the No-Lap population was predominantly characterised by elderly patients with poor performance status, severe comorbidities, hypoalbuminemia, elevated lactate, and creatinine levels, and those likely to have bowel ischemia at admission. These features also appeared to be common in the study by Mcllveen et al [12] and have also been described in patients who had undergone futile surgery despite extreme preoperative risk $[5,18,19]$. While all No-Lap patients but one died within 30-days after admission, early mortality within the first 30 days was also observed in the LAP cohort, however, to a lesser extent. A continuous increase in mortality was observed in the LAP cohort until 90 days after admission where the mortality seems to reach a steady state (Fig. 2). This may indicate potential variation in patient related characteristics and physiological adaptation to surgical stress response in late survivors vs. non-survivors.

The results of our study, and the results presented by Mcllveen et al indicate potential large differences in the complex procedure of pre-operative decision-making which may have an immense effect on patient outcomes [20]. Decision-making in emergency abdominal surgery is challenging due to a large heterogeneity in patient-related factors i.e. surgical pathology and physiology (perforation vs. intestinal obstruction, presence of frailty, comorbidities, and age)[6]. Other well-established factors affecting decision making include surgeons' clinical and operative experience, perception of risks and benefits of operative and non-operative treatment, external pressure to operate i.e. from patients or their relatives, and culture $[1,7,9,20,21]$.

The potential variation in pre-operative decision-making between our study and the study presented by Mcllveen et al seems to be large and raises several issues. Firstly, from an ethical point of view, if patients suitable for major emergency surgery are deselected from surgery due to cultural variations in health care systems, unnecessary excessive mortality can be expected. Secondly, these variations may hamper the development of evidence-based guidelines, as the comparison of research outcomes and evaluation of different patient care pathways are based on the operated patients, thus making it challenging to establish objective criteria to define surgical futility. Thirdly excessive surgery to patients with low life expectancy is futile and causes both unnecessary suffering and waste scarce surgical resources.

While the main strength of the current study is the prospective design with the inclusion of a high number of consecutive patients some limitations need to be addressed. Firstly, we conducted a single-center study which may limit the extrapolation of our findings, as different centers may have different demographic characteristics, center volume, and triage. Secondly, it is reasonable that the conduction of the study may have led to increased awareness about the No-LAP patients which may have led to different decision-making during the study period.

Moreover, the COVID-19 pandemic and the associated lockdowns have affected surgical health care services globally and should be considered as a potential limitation when interpreting the results of our 
COVID-19 may have had an impact on the incidence of patients presenting with major abdominal pathology- as some patients may have died at home/nursing home facilities instead of admission to surgical wards potentially biasing mortality outcomes. During the study period, there was a period with lockdown and cancellation of elective non-cancer surgery which may have led to fewer reoperations for complications during the study period. The average number of patients undergoing AHA surgery at our institution was 23,1 during the study period versus 26,4 during the same monthly period pre-Covid dates (15th October 2018-15th August2019), indicating a 14\% decrease. While this decrease could be due to reduced patient intake secondary to Covid-19 restrictions, it could also be due to fewer complications secondary to elective surgery. However, it should be noted that complications secondary to elective surgery have been shown to have lower mortality than primary emergency surgery[23]. During the COVID19 pandemic the hospital resources (Operating theathers for emergency surgery and Intensive care resources including bed availability) for AHA-patients were not affected. Finally, the lower number of patients undergoing surgery in the inclusion period could be due to an increase in the number of NoLap patients secondary to the present study, thus decreasing the number of patients presenting for surgery.

In the era of shared decision-making, proper identification of the frail surgical patient and early evaluation of whether surgical intervention is deemed futile, is of paramount importance, to avoid futile care which seems to be common[5]. Nevertheless, a complex clinical and ethical dilemma that every surgeon and anesthesiologist will face regularly, and probably face more frequently, as life expectancy and the need for emergency surgery is on the rise globally[24]. Preoperative decision-making must be a multidisciplinary assessment process where the surgeon and the anaesthesiologists address the morbidity and mortality risks associated with care concerning the patient's pathology, comorbidities, functional status, and wishes adequately to avoid futile care [25].

There is an obvious need for prospective multi-center studies to characterise the whole population of patients in whom surgery is indicated including the No-LAP population, and to establish a standardized report form for these decisions allowing comparisons to be made, and evidence-based guidelines for futility to be established, hopefully increasing quality of care for the patients.

Final results from an ongoing large multicenter, UK-based prospective cohort study aiming to characterize the No-LAP patients are awaited [26]. While this study may show potential regional and cultural differences between the centers in the UK it will not show potential cultural differences compared to other countries. This can be assessed by a prospective international multicenter cohort study in the future although it will require a standardization of cohort definitions and outcome measures.

Results from the UK-based multicenter study may however raise questions about which departments should manage these complex surgical patients if potential variation in decision making and mortalityrelated outcomes between low-volume and high volume surgical centers are found. [26]. Future studies should focus on objective assessment tools to identify patients in whom surgery may not be beneficial. 
Moreover, reporting of study outcomes including reporting outcomes for the No-LAP population should be standardized to make comparison feasible in future research.

\section{Conclusions}

There is an unmet need for prospective international multi-center studies to characterise the entire cohort of patients presenting with major pathology warranting acute high-risk abdominal surgery including the patients who do not proceed to surgery (No-LAP population), as large variations in triage criteria and culture probably exist and seem to impact outcome measures.

\section{Declarations}

Ethics approval and consent to participate: Permission for the data acquisition for the study was granted by the Center for Regional Development, Capital Region of Denmark (ID no. H-20036076) The Regional Ethics committee had no objections to the study (no. H-20036076) and did not require informed consent from the patients as the study was purely observational

Consent for publication: Not applicable.

Availability of data and materials: The datasets used and analysed during the current study are available from the corresponding author on reasonable request.

Competing interests: ME, MLL, MC, KL \& NB have no competing interests to declare.

Funding: The study received no external funding.

\section{Author contributions}

Study concept and design (MLL, NBF)

Acquisition of data (ME, MLL, MC, KLH)

Analysis and interpretation of data (ME, MLL, MC, KLH, NBF)

Drafting of the manuscript (ME, MLL, MC, KLH, NBF)

Critical revision of the manuscript for important intellectual content (ME, MLL, MC, KLH, NBF)

Statistical analysis (ME)

Study supervision (MLL, NBF)

All authors read and approved the final manuscript

Acknowledgements: Not applicable. 


\section{Authors' information:}

ME: Specialist Trainee in Surgery.

MLL: Senior consultant Emergency General Surgeon and Clinical Associate Professor.

MC: Intern, Ph.D. student.

KLH: Clinical research nurse.

NBF: Senior consultant in Anaesthesia and Professor.

\section{References}

1. Markar SR, Vidal-Diez A, Holt PJ, Karthikesalingam A, Hanna GB. An International Comparison of the Management of Gastrointestinal Surgical Emergencies in Octogenarians-England Versus United States: A National Population-based Cohort Study. Annals of surgery 2021; 273: 924-32.

2. Tengberg LT, Bay-Nielsen M, Bisgaard T, Cihoric M, Lauritsen ML, Foss NB; AHA study group. Multidisciplinary perioperative protocol in patients undergoing acute high-risk abdominal surgery. British Journal of Surgery 2017;104:463-71.

3. Vester-Andersen M, Lundstrom LH, Moller MH, Waldau T, Rosenberg J, Moller AM; Danish Anaesthesia Database. Mortality and postoperative care pathways after emergency gastrointestinal surgery in 2904 patients: A population-based cohort study. British Journal of Anaesthesia 2014; 112: 860-70.

4. Saunders DI, Murray D, Pichel AC, Varley S, Peden CJ; UK Emergency Laparotomy Network. Variations in mortality after emergency laparotomy: The first report of the UK emergency laparotomy network. British Journal of Anaesthesia 2012; 109: 368-75.

5. Chiu AS, Jean RA, Resio B, Pei KY. Early postoperative death in extreme-risk patients: A perspective on surgical futility. Surgery 2019; 166: 380-5.

6. Foss NB. Emergency laparotomy success - optimisation or triage? Anaesthesia 2020; 75: 1289-92.

7. Hendra L, Hendra T, Parker SJ. Decision-Making in the Emergency Laparotomy: A Mixed Methodology Study. World Journal of Surgery 2019; 43: 798-805.

8. Broughton KJ, Aldridge O, Pradhan S, Aitken RJ. The Perth Emergency Laparotomy Audit. ANZ Journal of Surgery 2017; 87: 893-7.

9. Nally DM, Sørensen J, Valentelyte $G$ et al. Volume and in-hospital mortality after emergency abdominal surgery: A national population-based study. BMJ Open 2019; 9:e032183.

10. Eugene N, Oliver CM, Bassett MG et al. Development and internal validation of a novel risk adjustment model for adult patients undergoing emergency laparotomy surgery: the National Emergency Laparotomy Audit risk model. British Journal of Anaesthesia 2018; 121: 739-48. 
11. Oliver CM, Walker E, Giannaris S, Grocott MPW, Moonesinghe SR. Risk assessment tools validated for patients undergoing emergency laparotomy: A systematic review. British Journal of Anaesthesia 2015; 115: 849-60.

12. Mcllveen EC, Wright $\mathrm{E}$, Shaw $\mathrm{M}$ et al. A prospective cohort study characterising patients declined emergency laparotomy: survival in the 'NoLap' population. Anaesthesia 2020; 75: 54-62.

13. von Elm E, Altman DG, Egger M, Pocock SJ, Gøtzsche PC, Vandenbroucke JP. The Strengthening the Reporting of Observational Studies in Epidemiology (STROBE) statement: guidelines for reporting observational studies. Lancet 2007; 370: 1453-7.

14. Gawande AA, Kwaan MR, Regenbogen SE, Lipsitz SA, Zinner MJ. An Apgar Score for Surgery. Journal of the American College of Surgeons 2007; 204: 201-8.

15. Harris PA, Taylor R, Minor BL et al. The REDCap consortium: Building an international community of software platform partners. Journal of Biomedical Informatics, 2019.

16. Oken MM, Creech RH, Davis TE. Toxicology and response criteria of the Eastern Cooperative Oncology Group. American Journal of Clinical Oncology: Cancer Clinical Trials, 1982;6:649-55

17. $\mathrm{R}$ Core Team (2020). R: A language and environment for statistical computing. $R$ : A language and environment for statistical computing. R Foundation for Statistical Computing, Vienna, Austria., 2020. .

18. Resio BJ, Chiu AS, Zhang Y, Pei KY. Characterization of High Mortality Probability Operations at National Surgical Quality Improvement Program Hospitals. JAMA Surgery 2020; 155: 85-8.

19. Aggarwal G, Broughton KJ, Williams LJ, Peden CJ, Quiney N. Early postoperative death in patients undergoing emergency high-risk surgery: Towards a better understanding of patients for whom surgery may not be beneficial. Journal of Clinical Medicine 2020; 9: 1-10.

20. Davis SS, Babidge WJ, McCulloch GAJ, Maddern GJ. Fatal flaws in clinical decision making. ANZ Journal of Surgery 2019; 89: 764-8.

21. Morris RS, Ruck JM, Conca-Cheng AM, Smith TJ, Carver TW, Johnston FM. Shared Decision-Making in Acute Surgical Illness: The Surgeon's Perspective. Journal of the American College of Surgeons 2018; 226: 784-95.

22. Nepogodiev D, Omar OM, Glasbey JC et al. Elective surgery cancellations due to the COVID-19 pandemic: global predictive modelling to inform surgical recovery plans. British Journal of Surgery 2020; 107: 1440-9.

23. Cihoric M, Tengberg LT, Foss NB, Gögenur I, Tolstrup M-B, Bay-Nielsen M. Functional performance and 30-day postoperative mortality after emergency laparotomy-a retrospective, multicenter, observational cohort study of 1084 patients. Perioperative Medicine 2020; 9: 1-11.

24. Torrance A, Powell S, Griffiths E. Emergency surgery in the elderly: challenges and solutions. Emergency Surgery 2015:7: 55-68.

25. Sivarajah V, Walsh U, Malietzis G, Kontovounisios C, Pandey V, Pellino G. The importance of discussing mortality risk prior to emergency laparotomy. Updates in Surgery 2020; 72: 859-65. 
26. Price A, Mclennan E, Boyle J, group on behalf of the E study. 535 ELF 2: DEFINING THE DENOMINATOR ELF STUDY GROUP. Age and Ageing 2021; 50: ii1-4.

\section{Figures}

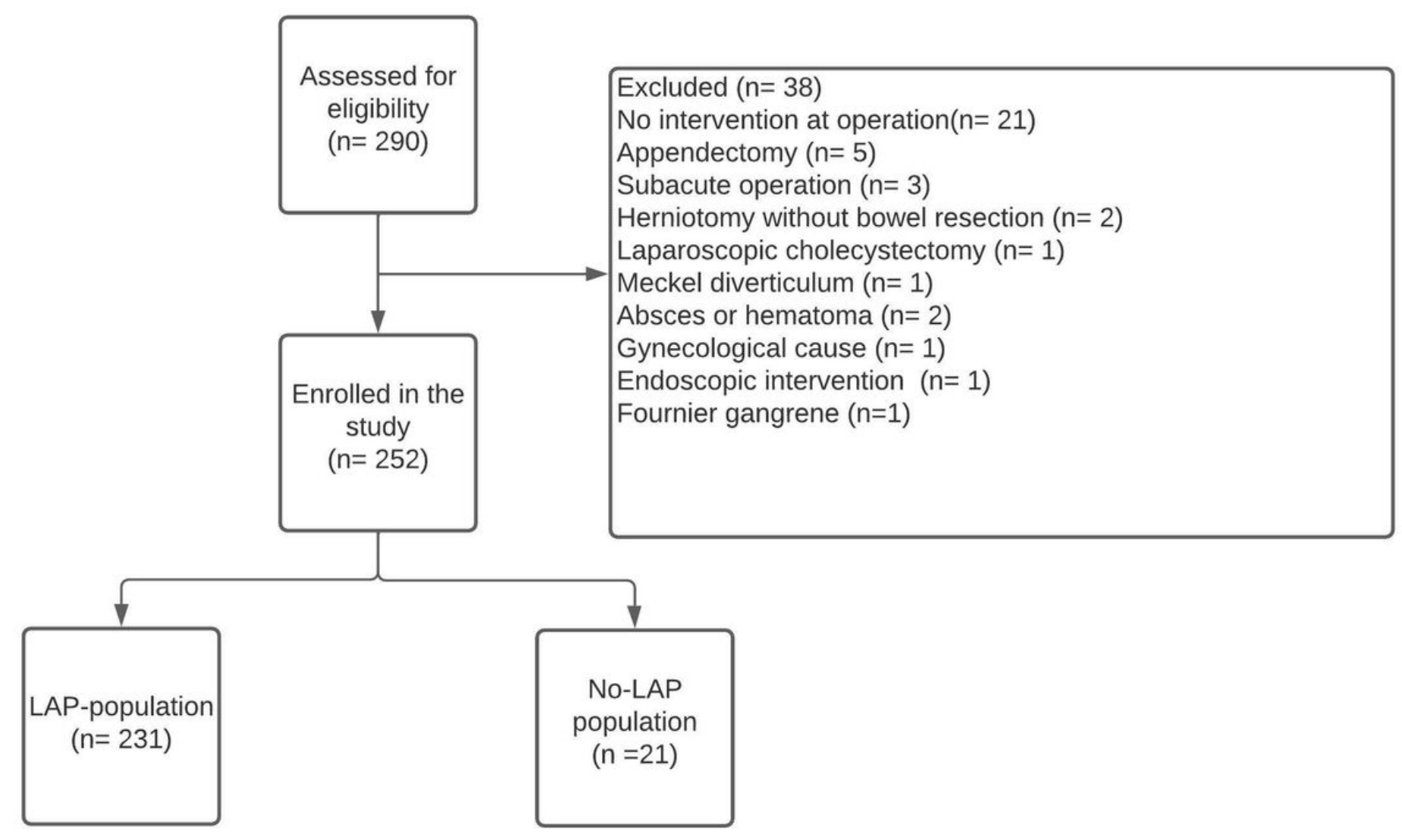

\section{Figure 1}

Flowchart of patients included in the present study. 


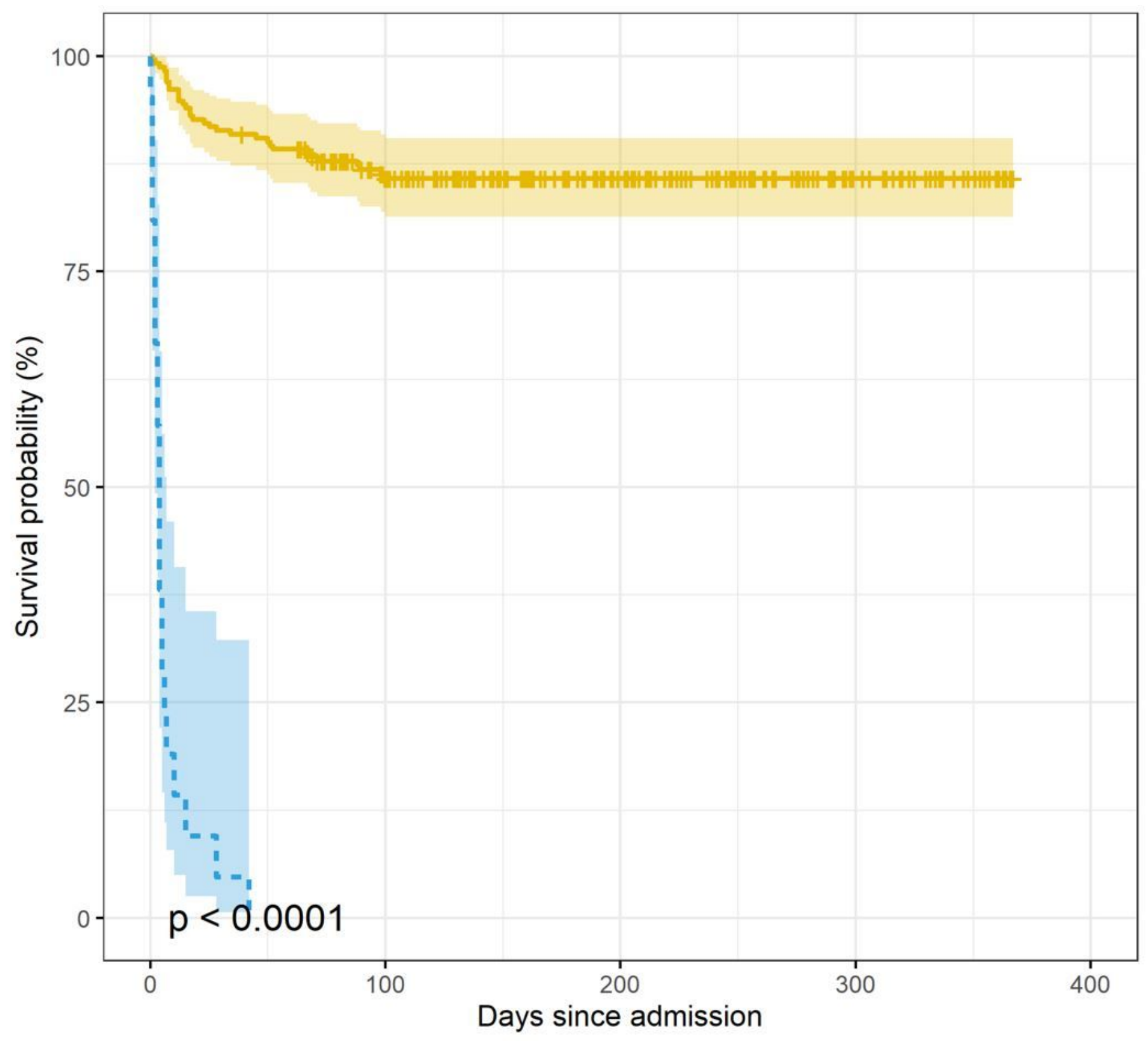

Strata + LAP + NOLAP

Figure 2

Kaplan-Meier survival curves showing survival probabilities for the LAP vs No-LAP group.

\section{Supplementary Files}

This is a list of supplementary files associated with this preprint. Click to download.

- OnlineSupportingInformationTableS1.docx 